\title{
BMJ Open Psychological distress of frontline healthcare workers in the intensive care unit during the early stage of the COVID-19 pandemic: a qualitative study from China
}

\author{
Zhao Yin (10 , ${ }^{1}$ Wan Zhang, ${ }^{1}$ Xuedong Jia, ${ }^{1}$ Xiaojuan Wang, ${ }^{1}$ Jie Hao (D) , 1 \\ Yantao Yang, ${ }^{1}$ Xiaojian Zhang, ${ }^{1}$ Shuzhang Du, ${ }^{1}$ Xiaohong Yue ${ }^{2}$
}

To cite: Yin Z, Zhang W, Jia X, et al. Psychological distress of frontline healthcare workers in the intensive care unit during the early stage of the COVID-19 pandemic: a qualitative study from China. BMJ Open 2022;12:e049627. doi:10.1136/ bmjopen-2021-049627

- Prepublication history for this paper is available online. To view these files, please visit the journal online (http://dx.doi. org/10.1136/bmjopen-2021049627).

Received 04 February 2021 Accepted 22 December 2021

D Check for updates

(C) Author(s) (or their employer(s)) 2022. Re-use permitted under CC BY-NC. No commercial re-use. See rights and permissions. Published by BMJ.

${ }^{1}$ Department of Pharmacy, The First Affiliated Hospital of Zhengzhou University, Zhengzhou, Henan, China ${ }^{2}$ Blood Purification Center, The First Affiliated Hospital of Zhengzhou University, Zhengzhou, Henan, China

Correspondence to Dr Xiaohong Yue; Yuexiaohong2009@126.com

\section{ABSTRACT}

Objective The rapid spread of COVID-19 has overwhelmed healthcare systems across the world. During the early stage of the pandemic, frontline healthcare workers (FHWs) caring for patients at intensive care units (ICUs) faced extreme pressure and challenges. This qualitative study aimed to describe the different phases of psychological distress of FHWs during the early stage of the COVID-19 pandemic.

Design Qualitative study.

Setting The First Affiliated Hospital of Zhengzhou University, a designated hospital for patients with COVID-19 in central China.

Participants Eight physicians and six nurses working in the ICU who provided direct patient care for COVID-19 patients.

Methods A descriptive phenomenological study using thematic analysis was applied. Semi-structured oneon-one interviews over telephone or Wechat (a social platform in China) rather than face-to-face interviews were conducted due to quarantine. Interviews were audiorecorded and transcribed verbatim and then were analysed thematically.

Findings $A$ total of 14 interviews were conducted, and each interview lasted 20-60 min. Five thematic categories were identified, and the participants' psychological experiences were classified into five stages (1) the mobilisation period: a sense of responsibility with worries; (2) the preparation period: worries, fears and doubts about the epidemic; (3) the transitional period: complex and diverse psychological feelings; (4) the adaptation period: self-adjustment and help from external support and (5) the reflection period: a reflection on life and nature.

Conclusion The study showed that the COVID-19 pandemic had significant psychological impacts on FHWs. Self-regulation and external support help FHWs to overcome challenges to a certain extent. More attention should be paid to the psychological wellbeing of ICU FHWs in COVID-19-designated hospitals.

\section{BACKGROUND}

Since the COVID-19 outbreak in early 2020, COVID-19 has become a global pandemic

\section{Strengths and limitations of this study}

- The present qualitative study contributes to understanding the psychological experience of frontline healthcare workers (FHWs) working in intensive care units during the early stage of the COVID-19 pandemic

- FHWs' psychological experiences during the early stage of the COVID-19 pandemic varied significantly. A qualitative study with in-depth interviews is necessary to understand their feelings.

- The findings may be limited to the geographical area and small study sample.

- The study sample might be limited in cultural backgrounds or religious beliefs, impacting the study findings.

and is showing no signs of coming to an end. ${ }^{1}$ As of 3 December 2021, there were 235 million confirmed cases, with 4.81 million deaths worldwide. Frontline healthcare workers (FHWs) are experiencing burnout and distress. ${ }^{2}$ The mental well-being of healthcare workers in fighting COVID-19 deserves our attention. Several studies have explored the impact of major pandemics and epidemics that occurred in the 21st century on the mental health of healthcare workers, such as SARS, the Middle East respiratory syndrome (MERS), Ebola virus disease (Ebola) and swine influenza. These studies found that most healthcare workers showed distressful psychological experiences during public health outbreaks, such as anxiety, stress, depression, post-traumatic stress disorder and burnout. Stress and anxiety were the most common feelings. ${ }^{3} 4$ A Saudi Arabian study reported the incidence rates among medical staff caring for patients with COVID-19 were $33.3 \%-68.5 \%$ for anxiety, 
$27.9 \%-55.2 \%$ for depression and $27.9 \%-68.5 \%$ for sleep disturbances and distress. ${ }^{5}$ These figures indicate that the COVID-19 pandemic is having severe psychological impacts on FHWs. A systematic review shows that the COVID-19 epidemic has affected the physical and mental health of FHWs, causing them to experience emotional distress such as fear, anxiety, depression and stress. In addition, the pandemic increases post-traumatic stress disorder, leading to burnout and interruption of medical work. ${ }^{6}$ A meta-analysis with 21 studies shows an urgent need for interventions to prevent or reduce the mental health problems of intensive care unit (ICU) medical staff caused by COVID-19. ${ }^{7}$ The results of two systematic reviews also indicate that nurses working on the frontline during the COVID-19 pandemic have experienced psychological, social and emotional distress in coping with work demands, social relationships and their personal lives. ${ }^{89}$

These results suggest that it is essential to provide necessary psychological interventions and social support for FHWs to relieve their related mental stress in their daily work of caring for patients with COVID19. At present, a variety of targeted government-level interventions have also been explored ${ }^{10} 11$ related to the mental well-being of healthcare workers. FHWs might have experienced unique psychological stress patterns at different operation stages, such as the mobilisation, preparation, transitional and adaptation periods. Taking more targeted measures for different stages of psychological development may improve the effectiveness of interventions. ${ }^{12}{ }^{13}$ However, there is no research report on the psychological development trajectory of FHWs. In this qualitative study, the healthcare workers' experiences and emotions engaged in fighting against COVID-19 were investigated to explore the course of psychological developments at different stages of the pandemic. The results may facilitate the development of interventional strategies corresponding to healthcare workers' psychological features in various stages and enhance the prevention and management of COVID-19.

\section{METHODS}

\section{Study design and setting}

The study was a descriptive phenomenological study with thematic analysis. Clinicians or nurses working in the ICU unit in a large COVID-19 designated hospital, the First Affiliated Hospital of Zhengzhou University, located in Zhengzhou, central China, were invited as participants. The hospital is one of the largest hospitals globally (with more than 8500 beds) and is the provincial designated hospital for COVID-19 patients in central China. Many critical-ill COVID-19 patients were treated in the ICU of this hospital, where physicians and nurses experienced heavy work pressures and psychological stress.

\section{Participants}

Purposeful sampling and snowball sampling methods were used to recruit participants. The invited participants had the experience of taking direct patient care for patients with COVID-19 for at least 2 weeks. Other inclusion criteria for participants are as follows: (1) working as full-time clinicians or nurses for at least 1 year in the sample hospital; (2) having at least a bachelor's degree; (3) willing to participate in the present study. For maximum variation in sampling, health workers of different professions were purposefully recruited. Enrolment was continued until data saturation was achieved.

\section{Data collection}

Semi-structured individual interviews were conducted over 2 months between March and April 2020 (the early stage of the COVID-19 pandemic in China). The interview time was determined based on the participant's availability. A quiet room was selected to ensure that the interview would be conducted without being disturbed. Telephone interviews were chosen since the participants were under quarantine when the research was done. Interviews generally lasted 20-60 min. Participants' demographic information was collected by emails or Wechat (a social networking platform in China). A team of nurses and pharmacists was established to oversee and conduct the research. Of note, all research members had experience in conducting qualitative studies.

An interview guide was developed based on the literature review, personal experience and expert opinion. A clinician and a nurse were invited to participate in a pilot interview and provide suggestions to revise the interview guide. The primary interview guide had four general questions: (1) why did you choose to accept this anti-epidemic task to work in the ICUs?; (2) what was the strongest feeling when working with the patients with COVID-19?; (3) what bothered you the most?; (4) what was the most stressful part of your work in the ICUs? These open-ended questions helped to probe into the participants' true feelings. Based on the responses, follow-up questions were asked to gain more insights into the answers. Modification of interview questions was done by the research team following each interview. The detailed interview guide with general and follow-up questions is shown in box 1 .

\section{Data analysis}

Thematic analysis was applied to understand the participants' perceptions. The interviews were recorded digitally and subsequently transcribed verbatim. The transcripts were reviewed by two research members ( $Z Y$ and WZ), checking for transcribing accuracy and consistency. All original recordings and transcriptions were in Chinese. The Chinese transcriptions were translated into English and back-translated into Chinese to ensure translation consistency. Data were analysed according 


\section{Box 1 Questions used in the interview guide}

\section{Q1: Why did you choose to accept this anti-epidemic task} to work in the ICUs?

- When you knew about the outbreak, medical staff needed to go to the frontline to treat patients with COVID-19. What did you think at the time?

- After you accepted the task, what was your strongest feeling at that time?

\section{Q2: What was the strongest feeling when working with the patients with COVID-19?}

- The previous interviewers mentioned that there would be some changes in their minds during the preparation period. What was your mentality at that time?

- In different periods of ICU work, such as when you just entered the ward, initially had contacts with patients, and in the middle of or the late stages of work, were your feelings different?

\section{Q3: What bothered you the most?}

- Was there any difference in your feelings at different stages of ICU work?

Q4: What was the most stressful part of your work in the ICUs?

- Was this stressful feeling the same at different stages? If it was not the same, could you give us a specific description?

ICU, intensive care unit.

to Haase's adaptation of the Colaizzi method. Two researchers (ZY and WZ) coded the interviews independently using the NVivo software (V.11). Extracting open topics, creating initial codes and drafting categories or subcategories were conducted in proper order. Representative quotations were selected by coders to present categories or subcategories. Discussions were carried out whenever there were disagreements in the coding process until consensus was reached. A senior research expert was invited to check and revise the extracted codes, categories and subcategories. The study was reported under the guidance of the Consolidated criteria for Reporting Qualitative research checklist.

\section{Trustworthy}

Trustworthiness was considered in terms of dependability, conformability, credibility and transferability. Accordingly, debriefing was done for each participant after the interview to improve credibility. Moreover, transferability was achieved by detailed data presentation from different study phases, considering the variations of participants. Finally, two researchers checked all manuscripts and memos to ensure data conformability.

\section{Patient and public involvement}

Patients and the public were not involved in the production of the present research.

\section{FINDINGS}

\section{Demographics}

Table 1 shows the demographic characteristics of the 14 participants (11 females), 8 physicians and 6 nurses. The average age was $33.60 \pm 7.34$ years, with work experiences ranging from 2 to 29 years $(9.14 \pm 8.08$ years). Five primary themes were identified from the interviews: (1) the mobilisation recruiting period: a strong sense of responsibility in the face of the epidemic; (2) the preparation period after accepting the task: worries, fears and doubts about the epidemic due to inadequate preparedness and uncertainty; (3) the transitional period at the early or middle phases of ICU care: complex and diverse psychological feelings; (4) the adaptation period during the middle or late phases of ICU care: self-adjustment and with help

Table 1 Baseline characteristics of the participants $(n=14)$

\begin{tabular}{llll}
\hline Number & DWBIs & Professional title & Department \\
\hline A1 & 22 & Chief physician & EICU \\
A2 & 22 & Surgeon-in-charge & EICU \\
A3 & 21 & Nurse-in-charge & Thoracic surgery \\
A4 & 22 & Chief nurse & PICU \\
A5 & 21 & Nurse-in-charge & PICU \\
A6 & 21 & Senior nurse & PICU \\
A7 & 22 & Chief physician & Respirology \\
A8 & 22 & Surgeon-in-charge & EICU \\
A9 & 22 & Surgeon-in-charge & EICU \\
A10 & 22 & Surgeon-in-charge & EICU \\
A11 & 21 & Primary nurse & PICU \\
A12 & 21 & Primary nurse & PICU \\
A13 & 14 & Clinician-in-charge & Cardiovascular \\
A14 & 14 & Clinician-in-charge & Haematology \\
\hline
\end{tabular}

DWBIs, days worked on COVID-19 ward before the interview (days); EICU, emergency intensive care unit; PICU, paediatric intensive care unit. 
from external support and (5) the reflection period after ICU patients' care.

Theme 1: mobilisation recruiting period—a sense of responsibility coupled with worries

Participants reported that fighting the epidemic was their job during the mobilisation period, with a positive sense of responsibility to rush to the frontline. Among them, young participants stated they should really step forward because they had gained enough clinical experience and their physical strength could fully meet the job demands. Meanwhile, some participants presented a sense of worry due to COVID-19.

\section{Subtheme 1.1: a sense of responsibility towards the nation}

The participants thought that many people were working hard for the future of their country. As healthcare providers, they should step in when the country is in an epidemic.

A4 (nurse): 'I think many people are working hard for the future of the country. Because this matter has developed to this stage, it is not a matter of one person or a certain person, but a matter of the entire Chinese people. No one can stay away from it, so everyone is working hard'.

\section{Subtheme 1.2: professional responsibility}

Some respondents felt it was their duty to contribute to the fight against the epidemic as medical workers.

A4 (nurse): 'I just thought we who are engaged in this profession need to do it'. A7 (clinician): 'As a respiratory clinician, I have relevant professional knowledge, so I should treat the patient on the frontline. That's it'.

\section{Subtheme 1.3: the responsibility of young professionals}

Young healthcare workers were more energetic and responsible in the face of the COVID-19 outbreaks, and they thought they had clinical expertise and were full of energy.

A11 (nurse): 'Well, I thought young people should rush to seize such an opportunity. I thought so at that time, so I signed up and came'.

\section{Subtheme 1.4: a sense of worries}

Participants expressed concern and worry at this stage.

A12 (nurse): 'When I heard about the recruitment of volunteers, I felt worried and even couldn't sleep!'

Theme 2: preparation period — worries, fears and doubts about the unknown

Most of the interviewees expressed that they became gradually nervous overtime during the preparation time. Participants stated that they were full of worries and fears about occupational exposure and personal protection in the isolation wards. Most of them were worried about the uncertainty of the situations in isolation wards or inadequate preparation.

\section{Subtheme 2.1: worries and fears}

After they were informed to work in the ICU isolation wards, some participants noted that feelings of worries and fears grew. Several nurses or physicians admitted they could not sleep well and eat well in the first few days when they accepted the mission.

A7 (clinician): 'I was a little afraid that the protection was not in place or I was exposed in the middle or something like that. I had some worries before I went in isolation wards'.

\section{Subtheme 2.2: doubt and anxiety}

The rapid and unpredictable development of the COVID-19 epidemic exerted tremendous psychological pressures on medical workers, leading to doubts and anxiety.

A14 (clinician): 'From the time the medical department informed me a few days ago until I went into ICU isolation wards, I was so nervous and anxious. Ha-ha, I didn't sleep well and eat well in a few days. I kept preparing for the frontline'. A4 (nurse): 'I was not sure, and I just felt that I didn't have enough time to prepare'.

Theme 3: transitional period—complex and diverse psychological feelings

When entering the isolation wards for the first time, ICU medical workers acted quickly to provide appropriate patient care. Their psychological characteristics were complex and diverse.

\section{Subtheme 3.1: fear}

The interviewees were primarily concerned about the unknown in the transitional period, whereas they turned to fear about the patient's serious illness in the initial stage of contact. Because of a lack of experience in dealing with such severe cases, they felt terrified.

A4 (nurse): 'We were dealing with severe cases. Sometimes we were terrified because some had never dealt with severe cases and seen such fearful scenes. Some might haven't even seen what had happened in the children's ICU wards before. They felt terrified and cried after work'.

\section{Subtheme 3.2: physical and mental exhaustion}

Due to various psychological pressures and highintensity workloads, interviewees experienced physical and mental exhaustion.

A2 (clinician): 'If you don't go to the battlefield or the frontline, you can never truly empathize .... After you come, you will find that it is extremely 
tiring, and it is not a physical overdraft but a kind of psychological suffering instead'.

\section{Subtheme 3.3: a sense of powerlessness and frustration}

The COVID-19 outbreak was a new disease, and health workers did not know how to deal with it. Clinicians and nurses felt frustrated when patients died.

A3 (nurse): 'The difficulty was that I felt at a loss in many areas. Because I didn't know how to deal with COVID-19, it felt like I couldn't use my strength in this case. I was helpless (sigh)'.

A9 (clinician): 'It was because we work in the ICU isolation wards, and the patients we contacted with are all very sick. It would be very uncomfortable to see the death of the critically ill patient'.

\section{Subtheme 3.4: adaptation}

As time went by, interviewees stated that they gradually became familiar with the work process and environment and adapted to work mentally.

A7 (clinician): 'Before we went in the isolation wards, we heard there were seven ventilators and six ECMOs, which were all for patients in serious condition. Well, of course, after going in now, we forgot our fear and the like when we got busy'.

Theme 4: adjustment and adaptation period—gradually building a positive mental state through self-adjustment and external support Subtheme 4.1: self-adjustment

Interviewees stated that they adapted to their role and adjusted their mentality. Participants reported that they coped with physical and mental stress through various methods, namely distraction, self-suggestion, dedication and the support of thirst for knowledge. Most of the interviewees expressed that focusing on patient care helped distract them from the negative mentality.

A7 (clinician): 'Well, I'd been observing the patients' indicators. Well, I adjusted the patients' parameters. In this case, I forgot the fear I had had'.

A3 (nurse): 'I mainly adjusted my state by selfsuggestion in the heart. Every time we rescued a patient, we felt depressed and bad in the heart, but we kept telling ourselves that I had done my best. I could do my best, and I have tried my best. However, we couldn't help with it, and we just gave ourselves psychological suggestions'.

A2 (clinician): 'Now I think air tube changing and intubation are so dangerous now, but I didn't have time to think about it at that time. I have a picture, and I found it after I did it. My head was about only five centimeters away from the patient's mouth. Later, I thought to myself that the distance of five centimeters was the distance between life and death.
Subtheme 4.2: external support

Positive media publicity, team cooperation, care from leaders and colleagues, and family support are all the spiritual strengths of medical staff.

Support of the public opinion-A3 (nurse): 'After work, I would check something positive on my cellphone, such as cure rates, some other reports, or some books to distract myself'.

Support of team strength-A3 (nurse): 'My biggest feeling is that the medical workers had an excellent tacit understanding. I think our team offered timely help'. Leadership concern-A9 (clinician): 'After getting out of the isolation ward, we communicated with leaders about these psychological barriers'. A10 (clinician): 'Then our leaders comforted us, 'anyway, you have paid a lot, and it is your greatest ability and the greatest ability of all of us.' I think it is beneficial to me and much needed'.

Support from colleagues-A1 (clinician): 'There is a cautious clinician. Everyone was grateful to her. Why? Because when we put on that overall, we would have difficulty bending down to wear the shoe cover. She squatted to help us wear the shoe cover'.

Support from family-A5 (nurse): 'I got the encouragement of my family. I went into the isolation wards right after weaning my baby. My family took care well of my baby'.

Theme 5: reflection period-re-recognise the value of life Subtheme 5.1: cherish life and nature

Subjects stated that after witnessing the death of critically ill patients and intuitively feeling the power of nature, they realised the need to cherish life and fear nature.

A6 (nurse): 'Well, cherish the present. (Smiling) It's better to live, and health is the most important, right?'

A1 (clinician): 'Nature is so magical that we can't bully the living things in nature randomly ... So we should protect them rather than hurt them at will'.

\section{Subtheme 5.2: cherish the present}

The respondents expressed that with the experience of working despairingly in the isolation ward and suffering the pain of separation from their families during the medical isolation, they realised the need to cherish the present.

A1 (clinician): 'Life is short. Ha-ha! I think sometimes we should cherish the present, which may be the biggest gain. Indeed, it is more important to cherish the present'.

A4 (nurse): 'Experience is to cherish life and live in the moment. Because we feel like we're walking away from life and death'.

\section{Subtheme 5.3: sublimate the value of life}

Many subjects reported channelling their life value through involvement in the frontline treatment. 
A4 (nurse): 'I think it is the most meaningful thing in my life to do something for my country for the first time'.

A14 (clinician): 'Yes, many people think this is a sublimation of life. Yes, yes, yes. ... I thought I would have been really sorry if I hadn't gone there.

\section{Subtheme 5.4: tempering individual wills}

Many interviewees mentioned that treating patients with COVID-19 in the ICU isolation ward was an unforgettable work experience, which tempered their wills and made them stronger in the heart.

A14 (clinician): 'I always thought I was so weak that I couldn't take some big responsibilities ... Young as I am in my family, I went to the frontline and could help others. Indeed, I have grown up a lot in terms of psychology. At least if I encounter some difficulties, I don't think I'll give up first'.

\section{DISCUSSION}

Our qualitative study revealed that FHWs experienced distinct psychological distress patterns when treating patients with COVID-19 during the early stage of the pandemic. The study findings contribute to a better understanding of the psychological experience of ICU healthcare providers.

The research results show that when mobilised to participate in the frontline treatment, medical staff show a strong sense of responsibility, including social and professional responsibility. Meanwhile, some interviewees also mentioned negative emotions such as worries. It is worth noting that the interviewees all voluntarily signed up to participate in treating patients with COVID-19 on the front line. Therefore, their negative emotions were relatively mild. ${ }^{14}$ However, after being notified to work in the frontline, most of them stated that they felt worried, fearful and doubtful about the pandemic. These negative emotions are primarily derived from the unknown information about COVID-19 and inadequate preparedness, consistent with the results of previous studies. A recently published systematic review mentioned that inadequate preparedness for and the unknown information about COVID-19 was the main reason for the accumulation of negative emotions. ${ }^{6}$ At the early stages of the COVID-19 pandemic, a study ${ }^{15}$ found that about one-third (342/994) of the healthcare providers in Wuhan, China had significant mental health disturbances. Among them, 6.2\% (62 of 994) had severe disturbances. ${ }^{15}$ For medical staff, the challenge brought by COVID-19 was mainly that they had limited time to prepare for working as FHWs during pandemic. ${ }^{16}$ This inadequate preparedness usually resulted in a situation where they had to work in crises, which led to an accumulation of negative emotions. ${ }^{1}$ Another major source of negative emotions among healthcare providers is the unknowns about COVID-19. A South Korean study reported that nurses had to work in a situation in which they were unfamiliar with the littleknown COVID-19 at the early stage of the pandemic. Most participants stated that they felt unprepared to work with patients with COVID-19, and mental health disturbances appeared shortly. ${ }^{17}$ In contrast, adequate preparation such as ideological preparation, professional psychological counselling, personal protection skills training, and COVID-19 patient care skills and knowledge training can help alleviate the negative psychological experience of FHWs, which deserves the attention of managers.

During the transitional period (halfway through the ICU assignment), FHWs experienced complex and various psychological disturbances, including fear, depression, exhaustion, frustration and powerlessness. ${ }^{18} \mathrm{~A}$ rapid systematic review reported that severe pandemics, such as SARS, Ebola, MERS and COVID-19 resulted in significant negative psychological impacts on healthcare providers. ${ }^{19}$ FHWs had tremendous psychological pressures and complex experiences. ${ }^{20}$ Most interviewees indicated that psychological burden, not physical fatigue, was the primary stressor in this study. During the pandemic, some medical workers were isolated from the public and their everyday lives. ${ }^{21}$ Previous studies assessed the common psychological impact of COVID-19 on healthcare providers. Among them, the most frequently reported psychiatric disorders diagnosed were depression, anxiety or post-trauma stress syndrome. ${ }^{22}$ Besides, somatization due to COVID-19 was reported frequently. Those somatic symptoms usually included headaches, throat pain, sleep disorders and lethargy. ${ }^{23}$ Participants from previous studies reported significant infection-related fears, such as fear of the unknown or becoming infected. ${ }^{24}$ An Italian study stated that the vulnerability of family members was also associated with worries or depression of FHWs. ${ }^{25}$ Participants in our study also feared that they might infect their family members. Therefore, it is essential to pay attention to the psychological impact of COVID-19 and other viral epidemics on FHWs.

During the adaptation period (mid to the end of ICU care), FHWs overcame various challenges through selfadjustment or external support. Our research did not find a precise separation time between the periods of transition and adaptation, and the timing of the turning point for each research subject was not consistent. The negative emotions that accumulated in prior stages still existed to some extent. HCWs' adjustment strategies mainly included distraction, self-suggestion or selfless dedication. In the past decade, many studies reported self-coping strategies during the epidemic outbreak. ${ }^{26}$ Behavioural disengagement, planning, self-distraction and exercises were commonly used as coping strategies. ${ }^{27}$ There are also many studies on external intervention strategies. ${ }^{19}$ Similar to our findings on external support, previous studies also highlighted the importance of support from social mechanisms, positive public news, the positive atmosphere of a team, support from group leaders or team members, and support from family members. ${ }^{28}$ A recent study claimed that psychological 
resources in the media or positive news help them through the epidemic. ${ }^{15}$ Correspondingly, support from supervisors and colleagues has been proven to be an effective way to overcome psychological burdens. ${ }^{29}$ A lack of external support had a negative psychological impact on FHWs.

We found that FHWs had a desire for skill training and related professional knowledge during the anti-pandemic period. Therefore, the emergency plan for public health events must be improved, and drills must be performed regularly. ${ }^{30}$ Building a good skill training programme and a relevant professional knowledge training system, while strengthening daily training, can help medical workers overcome panic psychology, improve their occupational protection effect and promote the success rate of clinical treatment. ${ }^{31}$ Literature suggests that medical workers involved in pandemic prevention and control were young and more inexperienced in service, having no experience related to severe acute respiratory syndrome. Most of them had not participated in disaster rescue knowledge training or simulation exercises, making them prone to panic and fear. ${ }^{32} 33$ Therefore, a sound public health emergency plan must be established at the government level. Various forms of emergency drills involving multiple departments must be performed at the social and hospital level. Additionally, daily training and assessment should be strengthened to improve the management process, communication mechanism and countermeasures.

There are, however, several limitations to the study: (1) although generalisability was not an intent, it is a limitation. The study covered a small number of participants in one hospital in China. Therefore, the findings may not represent healthcare workers in other culturally different contexts or areas of China, or other countries with different characteristics. Nevertheless, varied views were expressed; (2) social desirability bias is possible, although interviews were conducted by experienced, qualitative pharmacists' researchers to minimise this; (3) limitations commonly associated with semi-structured interviews apply to our study; (4) the interviews were not conducted in person so non-verbal behaviours or emotions could not be fully captured; and (5) the study involved two languages. Although during the translation process, efforts were made to increase validity and minimise the risk of losing meaning, there remains a risk that some meaning was lost in some cases.

\section{SUMMARY}

This study adds to the understanding of the psychological states of ICU medical workers treating patients with COVID-19 during the early stage of the pandemic. The identified themes help the medical society to assist the healthcare workers in future public health crises.

Contributors ZY, WZ and XY designed and performed the study, collected and analysed the data, and drafted the manuscript. XW helped collect the data. XJ, JH and $Y Y$ helped prepare the manuscript. $X Z$ and $S D$ revised the manuscript. $X Y$ is responsible for the overall content as the guarantor.
Funding The authors have not declared a specific grant for this research from any funding agency in the public, commercial or not-for-profit sectors.

Competing interests None declared.

Patient consent for publication Not required.

Ethics approval The study protocol was approved by the Institutional Review Board of the First Affiliated Hospital of Zhengzhou University (2020-KY-224). Written informed consent was obtained from all participants before the study started.

Provenance and peer review Not commissioned; externally peer reviewed.

Data availability statement Data are available upon reasonable request. The thematic data supporting this study's findings are available from the corresponding author on reasonable request.

Open access This is an open access article distributed in accordance with the Creative Commons Attribution Non Commercial (CC BY-NC 4.0) license, which permits others to distribute, remix, adapt, build upon this work non-commercially, and license their derivative works on different terms, provided the original work is properly cited, appropriate credit is given, any changes made indicated, and the use is non-commercial. See: http://creativecommons.org/licenses/by-nc/4.0/.

\section{ORCID IDs}

Zhao Yin http://orcid.org/0000-0003-2589-0962

Jie Hao http://orcid.org/0000-0001-7792-252X

\section{REFERENCES}

1 Lui RN, Wong SH, Sánchez-Luna SA, et al. Overview of guidance for endoscopy during the coronavirus disease 2019 pandemic. J Gastroenterol Hepatol 2020;35:749-59.

2 Duarte I, Teixeira A, Castro L, et al. Burnout among Portuguese healthcare workers during the COVID-19 pandemic. BMC Public Health 2020;20:1885.

3 Magill E, Siegel Z, Pike KM. The mental health of frontline health care providers during pandemics: a rapid review of the literature. Psychiatr Serv 2020;71:1260-9.

4 Fiest KM, Parsons Leigh J, Krewulak KD, et al. Experiences and management of physician psychological symptoms during infectious disease outbreaks: a rapid review. BMC Psychiatry 2021;21:91.

5 Bahamdan AS. Review of the Psychological Impact of COVID-19 Pandemic on Healthcare Workers in Saudi Arabia. Risk Manag Healthc Policy 2021;14:4105-11.

6 Koontalay A, Suksatan W, Prabsangob K, et al. Healthcare workers' burdens during the COVID-19 pandemic: a qualitative systematic review. J Multidiscip Healthc 2021;14:3015-25.

7 Troglio da Silva FC, Neto MLR. Psychiatric disorders in health professionals during the COVID-19 pandemic: A systematic review with meta-analysis. J Psychiatr Res 2021;140:474-87.

8 Lin Q, Zheng Y. Nurses' emotional stress levels when caring for COVID-19 patients in an intensive care unit. Altern Ther Health Med 2021;27:46-50.

9 Xu H, Stjernswärd S, Glasdam S. Psychosocial experiences of frontline nurses working in hospital-based settings during the COVID-19 pandemic - A qualitative systematic review. Int J Nurs Stud Adv 2021;3:100037.

10 Schwartz R, Sinskey JL, Anand U, et al. Addressing Postpandemic Clinician Mental Health: A Narrative Review and Conceptual Framework. Ann Intern Med 2020;173:981-8.

11 Jiménez-Giménez M, Sánchez-Escribano A, Figuero-Oltra MM, et al. Taking care of those who care: attending psychological needs of health workers in a hospital in Madrid (Spain) during the COVID-19 pandemic. Curr Psychiatry Rep 2021;23:44.

12 Azoulay E, De Waele J, Ferrer R, et al. Symptoms of burnout in intensive care unit specialists facing the COVID-19 outbreak. Ann Intensive Care 2020;10:110.

13 Brooks SK, Webster RK, Smith LE, et al. The psychological impact of quarantine and how to reduce it: rapid review of the evidence. Lancet 2020;395:912-20.

14 Roncone R, Giusti L, Mammarella S, et al. "Hang in There!": Menta Health in a Sample of the Italian Civil Protection Volunteers during the COVID-19 Health Emergency. Int J Environ Res Public Health 2021;18. doi:10.3390/ijerph18168587. [Epub ahead of print: 14 Aug 2021].

15 Kang L, Ma S, Chen M, et al. Impact on mental health and perceptions of psychological care among medical and nursing staff in Wuhan during the 2019 novel coronavirus disease outbreak: A cross-sectional study. Brain Behav Immun 2020;87:11-17. 
16 George CE, Inbaraj LR, Rajukutty S, et al. Challenges, experience and coping of health professionals in delivering healthcare in an urban slum in India during the first 40 days of COVID-19 crisis: a mixed method study. BMJ Open 2020;10:e042171.

17 Lee N, Lee H-J. South Korean Nurses' Experiences with Patient Care at a COVID-19-Designated Hospital: Growth after the Frontline Battle against an Infectious Disease Pandemic. Int J Environ Res Public Health 2020;17:9015.

18 Brooks SK, Greenberg N, Wessely S, et al. Factors affecting healthcare workers' compliance with social and behavioural infection control measures during emerging infectious disease outbreaks: rapid evidence review. BMJ Open 2021;11:e049857.

19 Cabarkapa S, Nadjidai SE, Murgier J, et al. The psychological impact of COVID-19 and other viral epidemics on frontline healthcare workers and ways to address it: A rapid systematic review. Brain Behav Immun Health 2020;8:100144.

20 Liu Q, Shen D, Chen S, et al. Supporting frontline nurses during the fight against COVID-19. J Am Psychiatr Nurses Assoc 2020;26:525-6.

21 Alizadeh A, Khankeh HR, Barati M, et al. Psychological distress among Iranian health-care providers exposed to coronavirus disease 2019 (COVID-19): a qualitative study. BMC Psychiatry 2020;20:494.

22 Liu C-Y, Yang Y-Z, Zhang X-M, et al. The prevalence and influencing factors in anxiety in medical workers fighting COVID-19 in China: a cross-sectional survey. Epidemiol Infect 2020;148:e98.

23 Chew NWS, Lee GKH, Tan BYQ, et al. A multinational, multicentre study on the psychological outcomes and associated physical symptoms amongst healthcare workers during COVID-19 outbreak. Brain Behav Immun 2020;88:559-65.

24 Balakrishnan V, Seong NK, Kaur W. COVID-19 Depression and Its Risk Factors in Asia Pacific - A Systematic Review and Metaanalysis. J Affect Disord 2021;18:S0165-0327:01267-2.
25 Rossi R, Socci V, Pacitti F, et al. Mental health outcomes among frontline and second-line health care workers during the coronavirus disease 2019 (COVID-19) pandemic in Italy. JAMA Netw Open 2020;3:e2010185.

26 Wong TW, Yau JKY, Chan CLW, et al. The psychological impact of severe acute respiratory syndrome outbreak on healthcare workers in emergency departments and how they cope. Eur J Emerg Med 2005;12:13-18

27 Korkmaz S, Kazgan A, Çekiç S, et al. The anxiety levels, quality of sleep and life and problem-solving skills in healthcare workers employed in COVID-19 services. J Clin Neurosci 2020;80:131-6.

28 Pan L, Xu Q, Kuang X, et al. Prevalence and factors associated with post-traumatic stress disorder in healthcare workers exposed to COVID-19 in Wuhan, China: a cross-sectional survey. BMC Psychiatry 2021;21:572.

29 Cai W, Lian B, Song X, et al. A cross-sectional study on mental health among health care workers during the outbreak of corona virus disease 2019. Asian J Psychiatr 2020;51:102111.

30 Catania G, Zanini M, Hayter M, et al. Lessons from Italian front-line nurses' experiences during the COVID-19 pandemic: a qualitative descriptive study. J Nurs Manag 2021;29:404-11.

31 Kotfis K, Williams Roberson S, Wilson JE, et al. COVID-19: ICU delirium management during SARS-CoV-2 pandemic. Crit Care 2020;24:176.

32 Zhang W-R, Wang K, Yin L, et al. Mental health and psychosocial problems of medical health workers during the COVID-19 epidemic in China. Psychother Psychosom 2020;89:242-50.

33 Fernández-Castillo R-J, González-Caro M-D, Fernández-García $\mathrm{E}$, et al. Intensive care nurses' experiences during the COVID-19 pandemic: a qualitative study. Nurs Crit Care 2021;26:397-406. 